\title{
CLASSIFYING SETS OF MEASURE ZERO WITH RESPECT TO THEIR OPEN COVERS
}

\author{
WINFRIED JUST AND CLAUDE LAFLAMME
}

\begin{abstract}
Developing ideas of Borel and Fréchet, we define a partial preorder which classifies measure zero sets of reals according to their open covers and study the induced partial order on the equivalence classes. The more "rarefied" a set of measure zero, the higher it will range in our partial order. Main results: The sets of strong measure zero form one equivalence class that is the maximum element of our order. There is a second highest class that contains all uncountable closed sets of measure zero. There is a minimum class that contains all dense $G_{\delta}$-subsets of the real line of measure zero. There exist at least four classes, and if Martin's axiom holds, then there are as many classes as subsets of the real line. It is also consistent with ZFC that there is a second lowest class.
\end{abstract}

\section{INTRODUCTION}

The definition of a cover of a subset $X$ of the unit interval used in this paper differs somewhat from the usual one. By cover of $X$ we always mean a sequence $\bar{I}=\left(I_{n}\right)_{n \in \mathbf{N}}$ of open intervals such that every $x \in X$ is contained in infinitely many of the intervals $I_{n}$.

The following idea was developed by Borel in Chapter 3 of his famous book [B]: Let $X, Y$ be subsets of the unit interval $[0,1]$, let $\bar{I}=\left(I_{n}\right)_{n \in \mathbf{N}}$ be a cover of $X$, and let $\bar{J}=\left(J_{n}\right)_{n \in \mathbf{N}}$ be a cover of $Y$. We say that $X$ is more rarefied than $Y$ iff $\lim _{n \rightarrow \infty}\left(\sum_{k \geq n}\left|I_{k}\right| / \sum_{k \geq n}\left|J_{k}\right|\right)=0$ (where $|I|$ denotes the length of the interval $I$ ).

In order to convert this idea into a precise mathematical concept one has to fix specific covers or to quantify over $\bar{I}$ and $\bar{J}$. A priori, there are four possible choices for quantifiers, and one might also wish to consider modifications like "not less rarefied" and

$$
\lim _{n \rightarrow \infty} \sup \left(\sum_{k \geq n}\left|I_{k}\right| / \sum_{k \geq n}\left|J_{k}\right|\right)<+\infty .
$$

Received by the editors November 7, 1988.

1980 Mathematics Subject Classification (1985 Revision). Primary 28A05; Secondary 03E35, 04A20.

Key words and phrases. Open cover, strong measure zero, ultrafilter.

Partially supported by an FCAR Postdoctoral research grant from the Province of Quebec (CL) and by NSERC grant A-7354 (WJ and CL). 
Some work has been done on different variants of the concept, but a detailed discussion of these lies beyond the scope of this paper. The interested reader should consult [F or Lf]. Instead, we propose our own variant and study the structure of sets of measure zero under the resulting partial preorder. This structure, or a least the part of it that we have been able to unravel, looks arbitrarily complicated if the continuum hypothesis or Martin's axiom (abbreviated $\mathrm{CH}$ and MA in the sequel) is assumed, but under different set-theoretical assumptions (related to Blass's NCF principle) it apparently can be of a remarkable simplicity and elegance.

To be sure, we cannot prove that our variant of the definition of "not less rarefied" is the "right" one, but the elegance of the emerging structure strongly indicates that it deserves further study.

The paper is organized as follows: In $\S 1$ we introduce the terminology and basic definitions used throughout the paper. Each of the subsequent sections is devoted to one theorem about the structure we investigate. The theorem itself is stated right at the beginning of each section. Moreover, prominent equivalence classes of measure zero sets are given proper names, and a picture illustrating the current state of knowledge accompanies each theorem. The last section contains a list of open questions.

\section{TERMINOLOGY AND BASIC DEFINITIONS}

We use standard set theoretic terminology. Functions $f \in 2^{\mathbf{N}}$ will be identified with points in the unit interval $[0,1]$; i.e., $f$ corresponds to the number $x_{f}=\sum_{n \in \mathrm{N}} f(n) 2^{-n-1}$. The characters $X$ and $Y$ always denote subsets of $[0,1]$ or $2^{\mathbf{N}}$ of Lebesgue measure zero. We think of $X$ as a subset of $[0,1]$ or $2^{\mathrm{N}}$ depending on what is more convenient for the given task. The intervals in $[0,1]$ correspond to the intervals in the lexicographic order on $2^{\mathbf{N}}$. We use $\mu$ for the measure on $[0,1]$ and $\lambda$ for the measure on $2^{\mathbf{N}}$. Since in all our considerations countable sets can be neglected, the fact that the mapping $f \rightarrow x_{f}$ is not one-to-one has no bearing.

Quantifiers $\forall_{n}^{\infty}, \exists_{n}^{\infty}$ mean "for all but finitely many $n$ " and "there exist infinitely many $n$ ", respectively.

Now we are ready to define the concept studied in this paper. Recall from $\S 0$ that $\bar{I}=\left(I_{n}\right)_{n \in \mathbf{N}}$ is a cover of $X$ iff $X \subseteq \bigcap_{n \in \mathbf{N}} \bigcup_{k \geq n} I_{k}$ and $\sum_{n}\left|I_{n}\right|<\infty$. Observe that a set $X$ has measure zero iff it has a cover.

Definition 1.1. We say that a set $X$ is at least as rarefied as a set $Y$ and write $X \geq Y$ if there is a nondecreasing function $r:(0,1) \rightarrow(0,1)$ such that for any cover $\bar{I}=\left(I_{k}\right)_{k \in \mathbf{N}}$ of $Y$, there is a cover $\bar{J}=\left(J_{k}\right)_{k \in \mathbf{N}}$ of $X$ such that $\forall_{n}^{\infty} r\left(\sum_{k \geq n}\left|J_{k}\right|\right) \leq \sum_{k \geq n}\left|I_{k}\right|$.

Note that by the definition of a cover, $\forall_{n}^{\infty}$ could be replaced by $\forall_{n}$. The relation $\leq$ is reflexive and transitive. We use the expressions "more rarefied than" $(>)$ and "less rarefied than" $(<)$ in their obvious sense. If $X \geq Y$ and 
$Y \geq X$, we say that $X$ and $Y$ are equivalent and write $X \sim Y$.

To illustrate the concept just introduced, consider Borel's definition of sets of strong measure zero. A set $X$ is of strong measure zero if for every sequence $\left(\varepsilon_{n}\right)_{n \in \mathbf{N}}$ of positive reals there exists a cover $\bar{I}=\left(I_{n}\right)_{n \in \mathbf{N}}$ of $X$ such that $\left|I_{n}\right|<\varepsilon_{n}$ for every $n$. It is not hard to see that if $X$ is of strong measure zero, and $Y$ is nonempty, then $X \geq Y$.

By $\mathbf{N} \uparrow \mathbf{N}$ we denote the family of nondecreasing unbounded functions from $\mathbf{N}$ to $\mathbf{N}$, and by $\mathbf{N} \downarrow(0,1)$ the family of nonincreasing sequences converging to zero. For a sequence $\bar{I}=\left(I_{n}\right)_{n \in \mathrm{N}}$ of nonempty intervals we define a function $f_{\bar{I}}$ by the formula $f_{\bar{I}}(n)=\left\lceil 1 / \sum_{k \geq n}\left|I_{K}\right|\right\rceil$.

For a set $X$ of measure zero we denote $\mathscr{M}(X)=\left\{f_{\bar{I}}: \bar{I}\right.$ is a cover of $\left.X\right\}$. If $f, g \in \mathbf{N} \uparrow \mathbf{N}$, we say that $f$ eventually dominates $g$ and write $f^{*} \geq g$ if $\forall_{n}^{\infty} f(n) \geq g(n)$. We say that a subfamily $\mathscr{M} \subset \mathbf{N} \uparrow \mathbf{N}$ dominates a family $\mathscr{N} \subset \mathbf{N} \uparrow \mathbf{N}$ if for every $g \in \mathscr{N}$ there exists an $f \in \mathscr{M}$ such that $g \leq^{*} f$. We say that $\mathscr{M} \subset \mathbf{N} \uparrow \mathbf{N}$ is dominating if $\mathscr{M}$ dominates $\mathbf{N} \uparrow \mathbf{N}$. We say that $\mathscr{M}$ is bounded if it is dominated by a singleton and call $\mathscr{M}$ unbounded otherwise. For $\mathscr{M} \subset \mathbf{N} \uparrow \mathbf{N}$ and $f \in \mathbf{N} \uparrow \mathbf{N}$ we denote $f \circ \mathscr{M}=\{f \circ g: g \in \mathscr{M}\}$.

The following proposition yields an alternative way to define the relation $\leq$.

Proposition 1.2. The following are equivalent:

(a) $X \geq Y$;

(b) $\exists f \in \mathbf{N} \uparrow \mathbf{N} f \circ \mathscr{M}(X)$ dominates $\mathscr{M}(Y)$.

Corollary 1.3. (a) If $X \geq Y$ and $\mathscr{M}(X)$ is bounded, then $\mathscr{M}(Y)$ is bounded.

(b) If $X \geq Y$ and $\mathscr{M}(Y)$ is dominating, then $\mathscr{M}(X)$ is dominating.

(c) If both $\mathscr{M}(X)$ and $\mathscr{M}(Y)$ are bounded, then $X \sim Y$.

We need some more terminology. A closed set $X$ is called self-supporting if $U \cap X$ is of positive measure for all open sets $U$ such that $U \cap X$ is nonempty.

Many of our examples will be constructed from ultrafilters. By $[\mathbf{N}]^{\omega}$ we denote the family of all infinite subsets of $\mathbf{N}$. We say that an infinite set $A$ is almost contained in a set $B$, and write $A \subseteq^{*} B$, if $A \backslash B$ is finite.

A subfamily $\mathscr{F} \subset[\mathbf{N}]^{\omega}$ is called a filter if for all $A, B \in[\mathbf{N}]^{\omega}$ :

If $A \in \mathscr{F}$ and $A \subseteq B$, then $B \in \mathscr{F}$.

If $A \in \mathscr{F}$ and $B \in \mathscr{F}$, then $A \cap B \in \mathscr{F}$.

A filter $\mathscr{F}$ is called an ultrafilter if $\forall A \in[\mathbf{N}]^{\omega} A \in \mathscr{F}$ or $\mathbf{N} \backslash A \in \mathscr{F}$. Ultrafilters will usually be denoted by letters $\mathscr{U}$ or $\mathscr{V}$. An ultrafilter $\mathscr{U}$ is called a $Q$-point if for every partition of $\mathbf{N}$ into pairwise disjoint intervals $\left[p_{i}, p_{i+1}\right]$, there is an $A \in \mathscr{U}$ such that $A \cap\left[p_{i}, p_{i+1}\right]$ has exactly one element for each $i \in \mathbf{N}$.

Two filters $\mathscr{G}$ and $\mathscr{F}$ are said to be cofinally equivalent [Bs] if there is a finite-to-one function $F: \mathbf{N} \rightarrow \mathbf{N}$ such that $F[\mathscr{G}]=F[\mathscr{F}]$, where $F[\mathscr{G}]=$ $\{F[A]: A \in \mathscr{G}\}$ and $F[A]=\{F(n): n \in A\}$. NCF is the statement asserting that every two ultrafilters are cofinally equivalent. 
A family $\mathscr{G} \subset[\mathbf{N}]^{\omega}$ is said to generate a filter $\mathscr{F}$ if

$$
\mathscr{F}=\left\{A \in[\mathrm{N}]^{\omega}: \exists B_{1}, B_{2}, \ldots, B_{n} \in \mathscr{G} B_{1} \cap B_{2} \cap \cdots \cap B_{n} \subseteq A\right\} .
$$

By $\underline{u}$ we denote the smallest cardinal of a family $\mathscr{G}$ that generates an ultrafilter.

A subfamily $\mathscr{D} \subseteq[\mathrm{N}]^{\omega}$ is said to be groupwise dense $[\mathrm{BL}]$ iff the following two conditions hold:

(a) If $A \in \mathscr{D}$ and $B \subseteq \subseteq^{*} A$, then $B \in \mathscr{D}$.

(b) If $\left(\left[p_{i}, p_{i+1}\right)\right)_{i \in \mathbf{N}}$ is a partition of $\mathbf{N}$ into pairwise disjoint intervals, then there exists an $A \in \mathscr{D}$ such that $A$ contains infinitely many of those intervals.

By $\underline{g}$ we denote the smallest cardinal $\kappa$ such that there exists a family $\left\{\mathscr{D}_{\xi}: \underline{\xi}<\kappa\right\}$ of groupwise dense subfamilies of $[\mathbf{N}]^{\omega}$ such that $\bigcap_{\xi<\kappa} \mathscr{D}_{\xi}=\varnothing$.

We mention two theorems about ultrafilters that will be used.

Theorem 1.4. If $C H$ or $M A$ holds, then there exist $2^{\underline{c}}$ Q-points that are pairwise not cofinally equivalent.

Theorem 1.5. The statement $\underline{u}<\underline{g}$ is relatively consistent with $Z F C$. Moreover, if $\underline{u}<\underline{g}$, then NCF holds.

The proof of Theorem 1.4 can be found in $[\mathrm{CN}]$; Theorem 1.5 was proved in $[\mathrm{BL}]$.

\section{SETS OF STRONG MEASURE ZERO}

Theorem 2.1. Suppose $X, Y$ are nonempty subsets of the unit interval and $X$ is of strong measure zero. Then $X \geq Y$. Moreover, $Y \geq X$ holds iff $Y$ is also of strong measure zero.

Let $\mathscr{S} \mathscr{M} \mathscr{Z}=\{X \subset[0,1]: X \neq \varnothing$ and $X$ is of strong measure zero $\}$. Theorem 1 gives Figure 1.

Lemma 2.2. Let $\varnothing \neq X \subset[0,1]$. Then the following are equivalent:

(a) $\mathscr{M}(X)$ is a dominating subfamily of $\mathbf{N} \uparrow \mathbf{N}$.

(b) $X$ is of strong measure zero.

Proof. Easy.

Theorem 2.1 is an immediate consequence of Lemma 2.2. and Corollary 1.3.

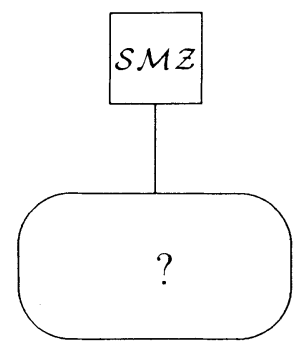

FIGURE 1 


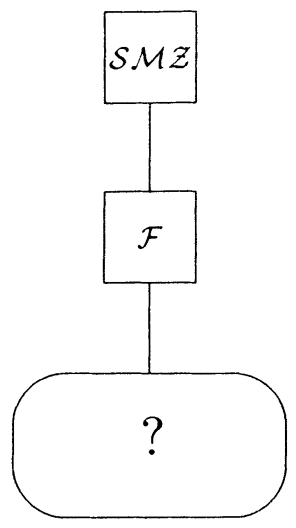

Figure 2

\section{THE SECOND HIGHEST CLASS}

Theorem 3.1. If $X$ is contained in an $F_{\sigma}$-set of measure zero and $Y$ is not of strong measure zero, then $X \geq Y$.

Since uncountable $F_{\sigma}$-sets are not of strong measure zero, it follows that there is an equivalence class $\mathscr{F}$ containing all uncountable $F_{\sigma}$-sets of measure zero and located as shown in Figure 2.

Definition 3.2. We say that $X \subset[0,1]$ has the $F$-property if there exists a function $g \in \mathbf{N} \downarrow(0,1)$ such that whenever a function $f \in \mathbf{N} \downarrow(0,1)$ satisfies $f(n) \geq g(n)$ for infinitely many $n$, there exists a cover $\bar{I}=\left(I_{n}\right)_{n \in \mathbf{N}}$ of $X$ such that $\sum_{k \geq n}\left|I_{k}\right| \leq f(n)$ for all $n$.

Theorem 3.1 is a consequence of the following two lemmas.

Lemma 3.3. If $X$ is contained in an $F_{\sigma}$-set of measure zero, then $X$ has the F-property.

Lemma 3.4. If $X$ has the F-property, and $Y$ is not of strong measure zero, then $X \geq Y$.

Proof of Lemma 3.3. Suppose $X \subset \bigcup_{m \in \mathbf{N}} \mathbf{F}_{m}$, where $\mathbf{F}_{m} \subseteq \mathbf{F}_{m+1}$ for all $n$, and the $\mathbf{F}_{n}$ 's are closed sets of measure zero. Let $\bar{J}=\left(J_{n}\right)_{n \in \mathbf{N}}$ be a cover of $X$. By compactness, there exists an increasing sequence $(n(m))_{m \in \mathbf{N}}$ such that $\mathbf{F}_{m} \subset J_{n(m)} \cup J_{n(m)+1} \cup \cdots \cup J_{n(m+1)-1}$ for every $m$.

Now let $g \in \mathbf{N} \downarrow(0,1)$ be such that $g(n(m+2)) \geq \sum_{k \geq n(m)}\left|J_{k}\right|$ for all $m$. We show that $g$ witnesses the $F$-property of $X$. Indeed, suppose $f \in$ $\mathbf{N} \downarrow(0,1)$ and $\left(n_{j}\right)_{j \in \mathbf{N}}$ is an increasing sequence such that $f\left(n_{j}\right) \geq g\left(n_{j}\right)$ for every $j$. For $j$, let $m_{j}$ be such that $n\left(m_{j}+1\right)<n_{j} \leq n\left(m_{j}+2\right)$. Without loss of generality we may assume that $m_{j}<m_{j+1}$ for every $j$.

Now we define a cover $\bar{I}$ of $X$ as follows:

$$
I_{n}= \begin{cases}J_{n} & \text { if } n\left(m_{j}\right) \leq n<n\left(m_{j}+1\right) \text { for some } j \\ \varnothing & \text { else }\end{cases}
$$




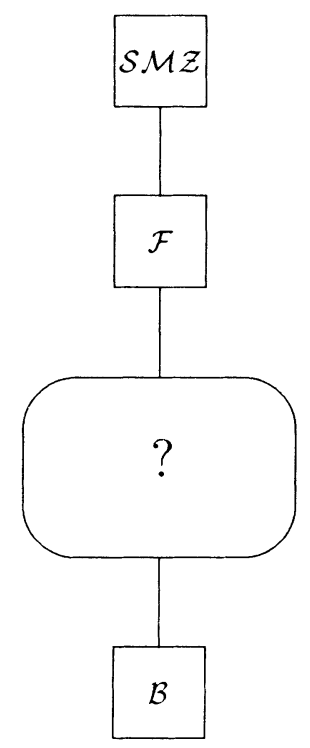

FIGURE 3

Now for arbitrary $n$ and for the smallest $j$ such that $n \leq n_{j}$ :

$$
\sum_{k \geq n}\left|I_{k}\right| \leq \sum_{k \geq n\left(m_{j}\right)}\left|J_{k}\right| \leq g\left(n\left(m_{j}+2\right)\right) \leq g\left(n_{j}\right) \leq f\left(n_{j}\right) \leq f(n) .
$$

Proof of Lemma 3.4. Suppose $g$ witnesses that $X$ has the $F$-property, and $Y$ is not of strong measure zero. Then there exists a function $h \in \mathbf{N} \downarrow(0,1)$ such that for every cover $\bar{J}=\left(J_{k}\right)_{k \in \mathbf{N}}$ of $Y$ the inequality $\sum_{k \geq n}\left|J_{k}\right| \geq h(n)$ holds for infinitely many $n$. Now it is not hard to find a nondecreasing function $r:(0,1) \rightarrow(0,1)$ such that $r \circ g(n) \leq h(n)$ for all $n$. The function $r$ as above witnesses that $X \geq Y$.

We conclude this section with the observation that the $F$-property actually characterizes the sets in $\mathscr{S} \mathscr{M} \mathscr{Z} \cup \mathscr{F}$.

Proposition 3.5. If $Y$ has the F-property, and $X \geq Y$, then $X$ has the $F$ property as well.

Proof. Suppose $g$ witnesses the $F$-property for $Y$, and let $r:(0,1) \rightarrow(0,1)$ witness $X \geq Y$. Without loss of generality we may assume that $r$ is $1-1$ and onto. We claim that $r^{-1} \circ g$ witnesses the $F$-property of $X$. Indeed, if $f$ is such that $r^{-1} \circ g(n) \leq f(n)$ for infinitely many $n$, then $g(n) \leq r \circ f(n)$ for these $n$, and hence there exists a cover $\bar{I}=\left(I_{n}\right)_{n \in \mathbf{N}}$ of $Y$ such that $\sum_{k \geq n}\left|I_{k}\right|<$ $r \circ f(n)$ for all $n$. By definition, there exists a cover $\bar{J}=\left(J_{n}\right)_{n \in \mathbf{N}}$ of $X$ such that $r\left(\sum_{k \geq n}\left|J_{k}\right|\right) \leq \sum_{k \geq n}\left|I_{k}\right| \leq r \circ f(n)$ for all $n$. It follows that $\sum_{k \geq n}\left|J_{k}\right| \leq f(n)$ for all $n$, as desired. 


\section{THE LOWEST CLASS}

Theorem 4.1. If a set $X$ of measure zero is such that there exists a self-supporting closed subset $V \subset 2^{\omega}$ of positive Lebesgue measure such that $V \backslash X$ is of first Baire category relative to $V$, then $X \leq Y$ for every $Y$ of measure zero.

It follows that at the bottom of the partial order there is an equivalence class $\mathscr{B}$ containing among others all comeager subsets of $[0,1]$ of measure zero.

In this section we concentrate on the proof of the following. (See Figure 3.)

Theorem 4.2. Suppose $X$ satisfies the hypothesis of Theorem 4.1. Then $\mathscr{M}(X)$ is bounded.

Theorem 4.1 follows from Theorem 4.2 by Corollary 1.3. We notice also that by Corollary 1.3, $X \in \mathscr{B}$ whenever $\mathscr{M}(X)$ is bounded.

Proof of Theorem 4.2. Let $X$ and $V$ be as in the hypothesis of Theorem 4.1. We denote $Y=V \backslash X$ and fix a sequence $\left(Y_{n}\right)_{n \in \mathbf{N}}$ of closed nowhere dense (in $V$ ) subsets of $V$ such that $Y_{n} \subset Y_{n+1}$ for all $n$, and $Y \subset \bigcup_{n} Y_{n}$.

Now we are going to define inductively sequences of natural numbers $(r(k))_{k \in \mathbf{N}}, \quad(b(k))_{k \in \mathbf{N}}$, and $(j(k))_{k \in \mathbf{N}}$ and a double sequence of finite functions $(s(i, k): k \in \mathbf{N}, 1 \leq i \leq j(k))$ such that for all $k \in \mathbf{N}$ :

(i) $r(k)<b(k)<r(k+1)$,

(ii) $b(k)-r(k) \geq k$ and $r(k+1)-b(k) \geq k$,

(iii) $s(1, k), \ldots, s(j(k), k) \in 2^{r(k)}$ are distinct. Equivalently, the sets $N_{s(1, k)}, \ldots, N_{s(j(k), k)}$ are pairwise disjoint, where $N_{s}=\left\{f \in 2^{\omega}\right.$ : $s \subset g\}$,

(iv) $\lambda\left(V \cap N_{s(i, k)}\right) \geq \frac{1}{2} \lambda\left(N_{s(i, k)}\right)=2^{-r(k)-1}$ for all $1 \leq i \leq j(k)$,

(v) If $s \in 2^{b(k)}$ and $N_{s} \cap V \neq \varnothing$, then there exists exactly one $i^{\prime}$ such that $s\left(i^{\prime}, k+1\right) \supset s$,

(vi) $N_{s(i, k)} \cap Y_{k}=\varnothing$ for $1 \leq i \leq j(k)$.

The construction can be carried out: Suppose we have already constructed $r(k)$ and the functions $s(i, k)$. Now we choose $b(k)$ large enough to satisfy the first half of (ii) and determine $j(k+1)$ to make (v) possible. Now we know $s(1, k+1) \uparrow b(k)$ for $1 \leq i \leq j(k+1)$ by the restrictions imposed by $(\mathrm{v})$. The $N_{s(i, k+1) i b(k)}$ 's are already pairwise disjoint. That will take care of (iii). We treat each of them separately. Since $Y_{k+1}$ is closed nowhere dense in $V$, we can extend $s(i, k+1)\left\lceil b(k)\right.$ to $\hat{s}(i, k+1)$ such that $Y_{k+1} \cap N_{\hat{s}(i, k+1)}=\varnothing$, and $N_{\hat{s}(i, k+1)} \cap V \neq \varnothing$.

Since $V$ is self-supporting, by the Lebesgue density theorem (see [O]), there exists an extension $\bar{s}(i, k+1)$ of $\hat{s}(i, k+1)$ such that $\lambda\left(V \cap N_{\bar{s}(i, k+1)}\right) \geq$ $\frac{1}{2} \lambda\left(N_{\bar{s}(i, k+1)}\right)$.

It is not hard to see that $\bar{s}(i, k+1)$ has extension $s(i, k+1)$ of arbitrary length such that (iv) holds. 
Now choose

$$
r(k+1)=\max \{b(k)+k, \operatorname{lh}(\bar{s}(1, k+1)), \ldots, \operatorname{lh}(\bar{s}(j(k+1), k+1))\},
$$

and extend each $\bar{s}(i, k+1)$ to $s(i, k+1)$ such that (iv) holds. This completes the construction.

It is worth noticing the following consequences of (i), (iv), and (v).

Fact 4.3. For very $m \leq k$ and for every $1<i<j(m)$ there are at least $2^{b(k)-r(m)-1}$ different extensions of the form $s\left(i^{\prime}, k+1\right)\lceil b(k)$ of $s(i, m)$.

Let $L(k)=\bigcup_{i=1}^{j(k)} N_{s(i, k)}$ for $k \in \mathbf{N}$, and denote $W=\left\{f \in 2^{\mathbf{N}}: \exists_{k}^{\infty} f \in\right.$ $L(k)\}$.

Proposition 4.4. $W \subset V \cap X$.

Proof. By (iv), $W \subset \operatorname{cl}(V)$, and since $V$ is closed, $W \subset V$. By (vi), $W \cap Y=$ $\varnothing$, so $W \subset X$.

Corollary 4.5. $W$ is of measure zero.

Lemma 4.6. $\mathscr{M}(W)$ and hence $\mathscr{M}(X)$ are bounded.

Proof. For $A \subset \omega$ infinite, we denote $W(A)=\left\{f \in 2^{\mathbf{N}}: \forall_{k \in A} f \in L(k)\right\}$. Clearly, $W(A) \subset W$. Lemma 4.6 is an immediate consequence of the following.

Sublemma 4.7. Let $A \subseteq \mathbf{N}$ be infinite. Assume $\bar{I}=\left(I_{n}\right)_{n \in \mathbf{N}}$ is a sequence of open intervals such that $\sum_{k \geq n}\left|I_{k}\right| \leq 2^{-r(n)-2}$ for all $n \in A$. Then $\bar{I}$ is not a cover of $W(A)$.

Proof of the sublemma. Let $(n(i))_{i \in \mathbf{N}}$ be an enumeration of $A \backslash\{0, \ldots, 6\}$ in increasing order. By assumption, we have

$$
\sum_{k=n(i)}^{n(i+1)-1}\left|I_{k}\right| \leq 2^{-r(n(i))-2} \text { for each } i \in \mathbf{N} .
$$

Using $(*)$, we construct $f \in W(A)$ such that $f \notin \bigcup_{k \geq n(0)} I_{k}$. We construct $f$ by induction. At each step $j$ of the construction we shall have

$$
\begin{gathered}
f(r(n(j))=s(i(j), n(j)) \text { for some } i(j) \text { and } \\
N_{f \mid r(n(j))} \cap \bigcap_{k=n(0)}^{n(j)-1} I_{k}=\varnothing .
\end{gathered}
$$

To begin with, let $f \mid r(n(0))=s(1, n(0))$. Now suppose $f\lceil r(n(j))$ has been defined so that $(\mathrm{j})$ holds. By Fact 4.3 , there are at least $2^{b(n(j+1)-1)-r(n(j))-1}$ pairwise disjoint subintervals of $N_{f \nmid r(n(j))}$, each of measure $2^{-b(n(j+1)-1)}$, where an extension of $f\lceil r(n(j))$ could go. The measure of the union of these intervals is at least $2^{-r(n(j))-1}$, and by $(*)$ at most one half of these are totally covered by $\bigcup_{k=n(j)}^{n(j+1)-1} I_{k}$. A further $2(n(j+1)-n(j))$ of them may have nonempty 
intersections with $\bigcup_{k=n(j)}^{n(j+1)-1} I_{k}$ (for this to happen, an interval must be cut be one of the edges of one of the $I_{k}$ 's).

It follows that there are at least $2^{b(n(j+1)-1-r(n(j))-2}-2(n(j+1)-n(j))=N$ possible extensions of $f \uparrow r(n(j))$ such that $(j+1)$ holds. Since $n(j+1)-1 \geq$ $n(j)$, we have, using condition (ii) of the construction, $b(n(j+1)-1)-r(n(j)) \geq$ $n(j+1)-1$, and hence $N>0$ since $n(0)>6$. So we always have a choice.

Remark 4.8. The requirement that $V$ is self-supporting cannot be dropped from the hypothesis of Theorem 4.1. To see this, let $Y \subset 2^{\omega}$ be an arbitrary nowhere dense closed set of positive Lebesgue measure. It is not hard to construct a family $\left\{V_{n}: n \in \mathbf{N}\right\}$ of pairwise disjoint basic open sets such that $\operatorname{cl}\left(\bigcup_{n} V_{n}\right) \subset Y \cup \bigcup_{n} V_{n}$, and for every open $U$ such that $U \cap Y \neq \varnothing$ there exists an $n$ such that $V_{n} \subset U$. Now choose nonempty closed sets $F_{n}$ of measure zero such that $F_{n} \subset V_{n}$ for every $n$. Then $\mathbf{F}=\bigcup_{n} F_{n}$ is comeager in $V=\mathbf{F} \cup Y$, but obviously $\mathbf{F} \in \mathscr{F}$ or $\mathscr{F} \in \mathscr{S} \mathscr{M} \mathscr{Z}$.

\section{THERE IS A FOURTH CLASS}

Theorem 5.1. There exists a set $X$ such that $\mathscr{M}(X)$ is unbounded and if $Y$ is a closed set of measure zero, then $Y>X$.

For reasons to becomes apparent below we choose to call the class containing $X$ as in Theorem 5.1 a " $\mathscr{U}$ "-class.

We get Figure 4.

Now it is time to reveal the machine that allowed us to produce almost all the examples discussed in the present paper.

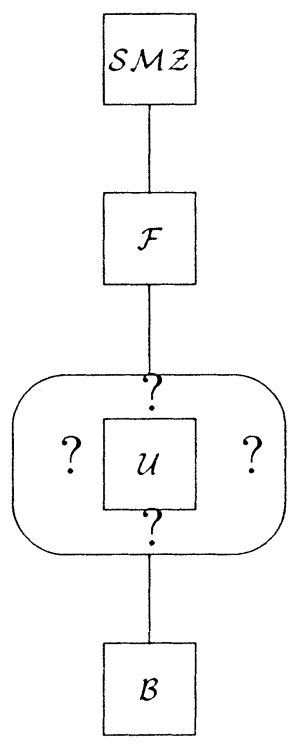

FIGURE 4 
Definition 5.2. (a) For $k \in \mathbf{N}, L_{k}=\left\{n \in \mathbf{N}: k^{2} \leq n<(k+1)^{2}\right\}$.

(b) For $\mathscr{A} \subset \mathscr{P}(\mathbf{N})$, denote

$$
\mathscr{S}(\mathscr{A})=\left\{f \in 2^{\mathbf{N}}: \exists A \in \mathscr{A} \forall k \in A f \uparrow L_{k} \equiv 0\right\} .
$$

(c) We write $\mathscr{S}(A)$ instead of $\mathscr{S}(\{A\})$ if $A \subseteq \mathbf{N}$.

Lemma 5.3. The set $\mathscr{S}\left([\mathbf{N}]^{\omega}\right)$ is of measure 0 .

Proof. Consider $2^{\mathbf{N}}$ as a probability space with the product measure. The probability $\operatorname{Pr}\left(\left\{f: f \mid L_{k} \equiv 0\right\}\right)=2^{-\left[(k+1)^{2}-k^{2}\right]}=2^{-2 k-1}$; hence the result follows from the Borel-Cantelli lemma.

Corollary 5.4. If $\mathscr{A}$ contains only infinite sets, then $\mathscr{S}(\mathscr{A})$ is of measure zero.

Notice that all classes isolated so far contain elements of the form $\mathscr{S}(\mathscr{A})$ :

If $\mathscr{A}$ consists only of cofinite sets, then $\mathscr{S}(\mathscr{A}) \in \mathscr{S} \mathscr{M} \mathscr{Z}$.

If $\mathscr{A}=\{A\}$ for some infinite, coinfinite $\mathscr{A}$, then $\mathscr{S}(\mathscr{A}) \in \mathscr{F}$.

If $\mathscr{A}=[\mathbf{N}]^{\omega}$, then $\mathscr{S}(\mathscr{A})$ is comeager in $2^{\mathbf{N}}$, and hence $\mathscr{S}(\mathscr{A}) \in \mathscr{B}$.

Definition 5.5. Let $\mathscr{A} \subset[\mathbf{N}]^{\omega}$.

(a) Following Mathias, we call $\mathscr{A}$ feeble iff there exists an increasing sequence $\left(p_{n}\right)_{n \in \mathbf{N}}$ of natural numbers such that $\forall A \in \mathscr{A} \forall_{n}^{\infty} \exists k \in A, p_{n} \leq k<p_{n+1}$.

(b) As usual, $\mathscr{A}$ is called linked iff $\forall A, B \in \mathscr{A}|A \cap B|=\aleph_{0}$.

(c) $\mathscr{A}$ is called spaced if $\forall A \in \mathscr{A} \quad \exists B \subset A \quad(B \in \mathscr{A} \& \forall a \in B \quad a+1 \notin B)$.

Example 5.6. (a) If $\mathscr{A}$ is a nonprincipal ultrafilter, then $\mathscr{A}$ is linked, nonfeeble, and spaced.

(b) If $\mathscr{A}$ is feeble, then $\mathscr{S}(\mathscr{A}) \in \mathscr{S} \mathscr{M} \mathscr{Z}$ or $\mathscr{S}(\mathscr{A}) \in \mathscr{F}$.

Theorem 5.1 is a corollary of Example 5.6(a) and the following.

Theorem 5.7. If $\mathscr{A}$ is linked, spaced, and nonfeeble, then $\mathscr{M}(\mathscr{S}(\mathscr{A}))$ is unbounded and $\mathscr{S}(\mathscr{A})$ is less rarefied then any closed subset of $2^{\mathbf{N}}$ of measure zero.

Proof of Theorem 5.7. Recall that for $s \in 2^{n}$ we denote $N_{s}=\left\{f \in 2^{\mathbf{N}}: f \uparrow n=\right.$ $s\}$. The concept defined below will be useful in several places.

Definition 5.8. Let $A=\{a(n): n \in \mathbf{N}\}$ be an infinite subset of $\mathbf{N}$. The canonical cover of $\mathscr{S}(\mathscr{A})$ is constructed as follows:

First list in lexicographical order the $2^{a(1)^{2}}$ intervals $I_{s}$ for $s \in 2^{(a(1)+1)^{2}}$ such that $s \uparrow L_{a(1)} \equiv 0$; then list in lexicographical order the $2^{a(2)^{2}}$ intervals $I_{s}$ for $s \in 2^{(a(2)+1)^{2}}$ such that $s \uparrow L_{a(2)} \equiv 0$; and so on through $A$.

Notice the important fact that $\left|I_{s}\right|=2^{-(n+1)^{2}}$ if $s \in 2^{(n+1)}$ and hence $\sum\left|I_{s}\right|=$ $2^{-2 n-1}$, where the sum is taken over all $s \in 2^{(n+1)^{2}}$ such that $s \uparrow L_{n} \equiv 0$.

Proposition 5.9. If $\mathscr{A}$ is linked and $A \in \mathscr{A}$, then the canonical cover of $\mathscr{S}(A)$ is a cover of $\mathscr{A}$. 
Lemma 5.10. Let $\mathscr{A}$ be linked and nonfeeble. For every sequence $\left(\varepsilon_{n}\right)_{n \in \mathbf{N}}$ of positive reals, there is a cover $\left(I_{n}\right)_{n \in \mathbf{N}}$ of $\mathscr{S}(\mathscr{A})$ such that $\sum_{k \leq n}\left|I_{k}\right|<\varepsilon_{n}$ for infinitely many $n$. In other words, $\mathscr{M}(\mathscr{S}(\mathscr{A}))$ is unbounded.

Proof. Fix the sequence $\left(\varepsilon_{n}\right)_{n \in \mathbf{N}}$ and assume without loss of generality that $\varepsilon_{n} \geq \varepsilon_{n+1}$ for each $n$.

Put $t(k)=\sum_{i \leq k} 2^{i^{2}}$ and define a sequence $(N(i))_{i \in \mathbf{N}}$ as follows: Put $n(0)=$ 0 and, having chosen $n(i)$, choose $n(i+1)$ large enough so that

$$
\sum_{n \geq n(i+1)} 2^{-2 n-1}<\varepsilon_{t(n(i))+1}
$$

Since $\mathscr{A}$ is nonfeeble, there exist an increasing sequence $\left(p_{j}\right)_{j \in \mathbf{N}}$ and a set $A \in \mathscr{A}$ such that $A \cap \bigcup_{j}\left[n\left(p_{j}\right), n\left(p_{j}+1\right)\right)=\varnothing$.

Now let $u(k)=\sum_{i \leq k, i \in A} 2^{i^{2}} \leq t(k)$, and let $\bar{I}=\left(I_{n}\right)_{n \in \mathbf{N}}$ be the canonical cover of $\mathscr{S}(A)$. Then for each $j$,

$$
\sum_{k \geq u\left(n\left(p_{j}\right)\right)+1}\left|I_{k}\right| \leq \sum_{n \geq n\left(p_{j}+1\right)} 2^{-2 n-1}<\varepsilon_{t\left(n\left(p_{j}\right)\right)+1} \leq \varepsilon_{u\left(n\left(p_{j}\right)\right)+1} .
$$

Since $\bar{I}$ is also a cover of $\mathscr{S}(\mathscr{A})$, this completes the proof of Lemma 5.10.

The next lemma parallels 4.6 and 4.7.

Lemma 5.11. Let $A$ be an infinite coinfinite subset of $\mathbf{N} \backslash\{0\}$. If $\bar{I}=\left(I_{k}\right)_{k \in \mathbf{N}}$ is a cover of $\mathscr{S}(A)$, then

$$
\sum_{k \geq a}\left|I_{k}\right| \geq 2^{-\operatorname{next}\left(A^{c}, a\right)^{2}-1}
$$

for infinitely many $a \in A$, where $\operatorname{next}\left(A^{c}, a\right)$ is the least element of the complement of $A$ greater than or equal to $a$.

Proof. Assume that $\bar{I}$ is such that for some $n, \forall a \in A \quad a \geq n \rightarrow \sum_{k \geq a}\left|I_{k}\right|<$ $2^{\text {next }\left(A^{c}, a\right)-1}$. Write $A \backslash n$ as $\bigcup_{i \in \mathrm{N}}[p(2 i), p(2 i+1))$, where $p(2 i+1) \notin A$. In particular:

$$
\sum_{k=p(2 i)}^{p(2 i+2)-1}\left|I_{k}\right|<2^{-p(2 i+1)^{2}-1} \quad \text { for each } i \in \mathbf{N} .
$$

Using (*) we build an $f \in \mathscr{S}(A)$ such that $f \notin \bigcup_{k \geq p(0)} I_{k}$ and hence $\bar{I}$ is not a cover of $\mathscr{S}(A)$. The function $f$ is constructed inductively such that at each stage $j$, we have

$$
N_{f \nmid p(2 j)^{2}} \cap \bigcup_{k=p(0)}^{p(2 j)-1} I_{k}=\varnothing .
$$

First put $f \mid p(0)^{2} \equiv 0$. Now assume $f \nmid p(2 j)^{2}$ has been defined and satisfies (j); extend $f$ to be identically zero on $L_{k}$ for $p(2 j) \leq k<p(2 j+1)$ 
(this will make sure that $f \in \mathscr{S}(A)$ ). We want to further extend $f$ to $p(2 j+2)^{2}$ so that $(j+1)$ holds. But $\bigcup_{k=p(2 j)}^{p(2 j+2)-1} I_{k} \cap N_{f\left\lceil p(2 j+2)^{2}\right.}=\varnothing$ for at least $N=$ $2^{p(2 j+2)^{2}-p(2 j+1)^{2}-1}-2(p(2 j+2)-p(2 j))$ choices of $f^{\prime} \uparrow(n(2 j+2))^{2}$ extending the part of $f$ previously defined. Hence $N>0$ and $f \uparrow(p(2 j+2))^{2}$ can be defined so as to satisfy $(j+1)$. This completes the proof.

Lemma 5.12. Let $X \subset 2^{\mathbf{N}}$, and let $\mathscr{A} \subset \mathscr{P}(\mathbf{N})$ be spaced. Assume that for each sequence $\left(\varepsilon_{n}\right)_{n \in \mathbf{N}}$ of positive reals there are $A=\left\{a_{n}: n \in \mathbf{N}\right\} \in \mathscr{A}$ and a cover $\bar{I}=\left(I_{n}\right)_{n \in \mathbf{N}}$ of $X$ such that

$$
\forall n \quad \sum_{k \geq a_{n}}\left|I_{k}\right| \leq \varepsilon_{a_{n}}
$$

Then $\mathscr{S}(\mathscr{A}) ¥ X$.

Proof. We need to show that for each nondecreasing function $r:(0,1) \rightarrow$ $(0,1)$, there is a cover $\bar{I}=\left(I_{n}\right)_{n \in \mathbf{N}}$ of $X$ such that for every cover $\bar{J}=\left(J_{n}\right)_{n \in \mathbf{N}}$ of $\mathscr{S}(\mathscr{A})$ :

$$
r\left(\sum_{k \geq n}\left|J_{k}\right|\right)<\sum_{k \geq n}\left|I_{k}\right| \text { holds for infinitely many } n .
$$

Fix $r$ and assume without loss of generality that $r(x) \leq x$ for all $x$. Define a sequence $\left(\varepsilon_{n}\right)_{n \in \mathbf{N}}$ of positive reals such that

$$
\forall x \in(0,1) \forall n \in \mathbf{N} \quad r(x)<\varepsilon_{n} \rightarrow x<2^{-(n+1)^{2}-1} .
$$

Now choose $A=\{a(n): n \in \mathbf{N}\} \in \mathscr{A}$ and a cover $\bar{I}=\left(I_{n}\right)_{n \in \mathbf{N}}$ of $X$ witnessing $(*)$. Since $\mathscr{A}$ is spaced, we may without loss of generality assume that $a(n)+1<a(n+1)$ for all $n$. Fix an cover $\bar{J}=\left(J_{n}\right)_{n \in \mathbf{N}}$ of $\mathscr{S}(\mathscr{A})$. If (**) fails, then there exists an $m \in \mathbf{N}$ such that $r\left(\sum_{k \geq n}\left|J_{k}\right|\right) \leq \sum_{k \geq n}\left|I_{k}\right|$ for all $n \geq m$, and hence in particular $r\left(\sum_{k \geq a(n)}\left|J_{k}\right|\right) \leq \sum_{k \geq a(n)}\left|I_{k}\right| \leq \varepsilon_{a(n)}$.

By construction we get $\sum_{k>a(n)}\left|J_{k}\right| \leq 2^{-(a(n)+1)^{2}-1}$ for each $a(n) \geq m$. By Lemma $5.11, \bar{J}$ is not a cover of $\mathscr{S}(A)$. Since $\mathscr{S}(A) \subseteq \mathscr{S}(\mathscr{A})$, we have obtained a contradiction and the proof of the lemma is complete.

To finish the proof of Theorem 5.7, fix any sequence $\left(\varepsilon_{n}\right)_{n \in \mathbf{N}}$ of positive reals, assume without loss of generality that $\varepsilon_{n} \geq \varepsilon_{n+1}$ for all $n$, and fix a closed set $X$ and a cover $\bar{J}=\left(J_{n}\right)_{n \in \mathbf{N}}$ of $X$.

By the compactness of $X$, we find an increasing sequence $(k(i))_{i \in \mathbf{N}}$ such that for each $i$ :

(1) $X \subseteq \bigcup_{n=k(i)}^{k(i+1)-1} J_{n}$.

(2) $\sum_{k \geq k(i+1)}\left|J_{k}\right| \leq \varepsilon_{k(i)}$.

Since $\mathscr{A}$ is nonfeeble, there exist $A \in \mathscr{A}$ and an infinite sequence $(j(m))_{m \in \mathbf{N}}$ such that $j(m)+2<j(m+1)$ and $A \cap \bigcup_{m \in \mathbf{N}}[k(j(m)), k(j(m)+2))=\varnothing$. Define 
a cover $\bar{I}=\left(I_{n}\right)_{n \in \mathbf{N}}$ of $X$ by

$$
I_{n}= \begin{cases}j_{n} & \text { if } \left.n \in \bigcup_{m}[k(j(m)+1), k(j(m)+2))\right), \\ \varnothing & \text { otherwise. }\end{cases}
$$

Then for each $a \in A$, say $a \in[k(j(m-1)+2), k(j(m)))$,

$$
\sum_{k \geq a}\left|I_{k}\right| \leq \sum_{n \geq k(j(m)+1)}\left|J_{n}\right| \leq \sum_{k(j(m))} \leq \varepsilon_{a} .
$$

Therefore, $A$ satisfies the hypothesis of Lemma 5.12 and we obtain $\mathscr{S}(\mathscr{A}) \nsupseteq$ $X$. By 2.1 and $3.1, X \geq \mathscr{S}(\mathscr{A})$, and the proof is now complete.

\section{IF $\underline{u}<\underline{g}$, A CLEAN PICTURE EMERGES}

Theorem 6.1. Suppose $\underline{u}<\underline{g}$. Then there exists an equivalence class $\mathscr{U}$ of the relation $\sim$ which contains all sets of the form $\mathscr{S}(\mathscr{V})$ where $\mathscr{V}$ is a nonprincipal ultrafilter on $\mathbf{N}$ and such that whenever $X \in \mathscr{U}$ and $Y$ is of measure zero, then $Y \in \mathscr{B}$ or $Y \geq X$.

Moreover, if $\mathscr{A}$ is any family of infinite subsets of $\mathbf{N}$, then $\mathscr{S}(\mathscr{A})$ belongs to one of the four classes $\mathscr{S} \mathscr{M} \mathscr{Z}, \mathscr{F}, \mathscr{U}, \mathscr{B}$.

Figure 5 emerges.

We now need some terminology. In this section we shall write $\mathscr{M}(\mathscr{A})$ as shorthand for $\mathscr{M}(\mathscr{S}(\mathscr{A}))$. For a set $A \in[\mathbf{N}]^{\omega}$ and $n \in \mathbf{N}$ we denote by $\operatorname{next}(A, n)$ the least element of $A$ not less than $n$.

Lemma 6.2. Let $\mathscr{U} \subset[\mathbf{N}]^{\omega}$ be an ultrafilter generated by the family $\{A(\alpha): \alpha<$ $\underline{u}\}$, which we assume closed under finite intersections. Then there is a family of functions $\mathscr{D}=\left\{h_{\alpha}: \alpha<\underline{u}\right\} \subset \mathbf{N} \uparrow \mathbf{N}$ such that $\mathscr{D}$ dominates $\mathscr{M}(\mathscr{U})$.

Proof. Define $h_{\alpha}(n)=2^{[\operatorname{next}(A(\alpha), n)+1)]^{2}+1}$. We show this works. Let $f \in \mathscr{M}(\mathscr{U})$ and $\bar{I}=\left(I_{n}\right)_{n \in \mathbf{N}}$ be a cover of $\mathscr{S}(\mathscr{U})$ such that $f=f_{\bar{I}}$. By Lemma 5.11 we can find some $\alpha<\underline{u}$ such that $f(x)=\left\lceil 1 / \sum_{k \geq x}\left|I_{k}\right|\right\rceil \leq 2^{(x+1)^{2}+1}$ for every $x \in A(\alpha)$.

Now by the choice of $h_{\alpha}$, we have for each $n$

$$
\begin{aligned}
f(n) & =\left\lceil 1 / \sum_{k \geq n}\left|I_{k}\right|\right\rceil \leq\left\lceil 1 / \sum_{k \geq \operatorname{next}(A(\alpha), n)}\left|I_{k}\right|\right\rceil \\
& \leq 2^{\operatorname{next}(A(\alpha), n)+1]^{2}+1}=h_{\alpha}(n) .
\end{aligned}
$$

Lemma 6.3. Suppose $X$ is of measure zero, but not in the $\mathscr{B}$ class, and $\mathscr{M}(Y)$ is dominated by a set $\mathscr{D}$ of size less than $g$. Then $X \geq Y$.

Proof. Suppose $\kappa<g$, and $\mathscr{D}=\left\{h_{\alpha}: \alpha<\kappa\right\}$ dominates $\mathscr{M}(Y)$. Define

$T_{\alpha}=\left\{B \in[\mathbf{N}]^{\omega}:\right.$ for some $f \in \mathscr{M}(X)$, the inequality next $(B, f(n))$ $\geq h_{\alpha}(n)$ holds for all but finitely many $\left.n\right\}$. 

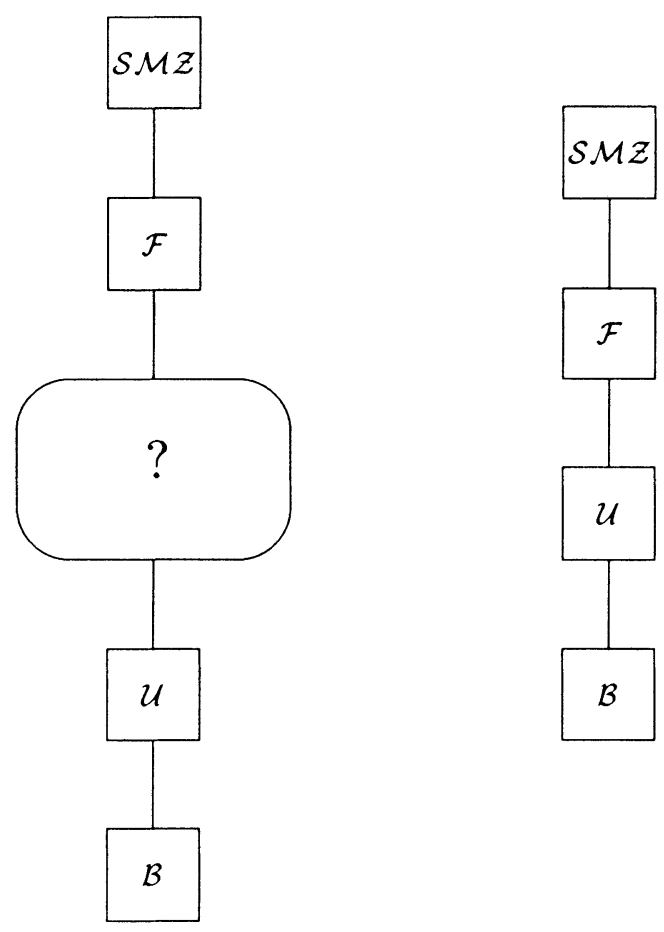

\section{FigURE 5}

Claim 6.4. Each set $T_{\alpha}$ is groupwise dense.

Proof of the claim. Clearly, each set $T_{\alpha}$ is closed under subsets and finite modifications. Now fix a partition of $\mathbf{N}$ into intervals $\left[p_{i}, p_{i+1}\right)$. We show that some union of infinitely many of those intervals is in $T_{\alpha}$. We assume without loss of generality that $h_{\alpha}\left(p_{i}\right)<p_{i+1}$ for all $i$ (otherwise merge enough intervals together).

Now fix $g \in \mathbf{N} \uparrow \mathbf{N}$ such that $g\left(p_{i}\right) \geq p_{i+4}$ for all $i$, and since $\mathscr{M}(X)$ is unbounded, find $f \in \mathscr{M}(X)$ such that $f(n) \geq g(n)$ for infinitely many $n$. Put $B=\bigcup\left\{\left[p_{i+2}, p_{i+3}\right): \exists n \in\left[p_{i}, p_{i+1}\right) \quad f(n) \geq g(n)\right\}$. We show that $B \in T_{\alpha}$, i.e., that $\operatorname{next}(n, f(m)) \geq h(m)$ for all $m$. Find $m$, say $m \in\left[p_{i}, p_{i+1}\right)$, and let $y=\operatorname{next}(B, f(m))$, say $y \in\left[p_{j+2}, p_{j+3}\right)$.

Notice that the inequality $f(m)<m$ may hold, but we have

Subclaim 6.5. $y \geq m$, and therefore $j+2 \geq i$.

Proof of the subclaim. Assume that $y<m$, and hence $f(m) \leq y<m$. By definition of $B$, there is $n \in\left[p_{j}, p_{j+1}\right)$ such that $f(n) \geq g(n)$ and hence $f(n) \geq g(n) \geq p_{j+4}>y \geq f(m)$. But $n<y<m$, and hence $f(n) \leq f(m)$, since $f \in \mathbf{N} \uparrow \mathbf{N}$. This contradiction completes the proof of the subclaim. 
Now, if $j \geq i$, then $y \geq p_{i+2} \geq h(m)$, and we are done. If $j<i$, then again choose $n \in\left[p_{j}, p_{j+1}\right)$ such that $f(n) \geq g(n) \geq p_{j+4}$. Since $j+1 \leq i$, we have $n \leq m$, and hence $y \geq f(m) \geq f(n) \geq p_{j+4} \geq p_{i+2} \geq h_{\alpha}(m)$.

This completes the proof of the claim.

Now since each $T$ is groupwise dense, and there are fewer than $\underline{g}$ of these sets, their intersection is nonempty.

Let $B \in \bigcap_{\alpha<\kappa} T_{\alpha}$, and define a function $r:(0,1) \rightarrow(0,1)$ by $r(x)=$ $[\operatorname{next}(B,\lceil 1 / x\rceil)]^{-1}$. This function witnesses that $X \geq Y$ : If $\bar{I}=\left(I_{n}\right)_{n \in \mathbf{N}}$ is a cover of $Y$, then $f_{\bar{I}}$ is dominated by some $h_{\alpha}$, and since $B \in T_{\alpha}$, there exists a cover $\bar{J}=\left(J_{n}\right)_{n \in \mathbf{N}}$ such that $\operatorname{next}\left(B, f_{\bar{J}}\right) \geq h_{\alpha}(n)$ for all but finitely many $n$. Now by the definition of $r$, this implies that $r\left(\sum_{k \geq n}\left|J_{k}\right|\right) \leq \sum_{k \geq n}\left|I_{k}\right|$ for almost all $n$.

Corollary 6.6. If $\underline{u}<\underline{g}$ then there exists an ultrafilter $\mathscr{U}$ such that $\mathscr{S}(\mathscr{U}) \leq X$ for every set $X$ of measure zero not in $\mathscr{B}$.

The following proposition is the last brick in the proof of Theorem 6.1.

Proposition 6.7. Suppose $\underline{u}<\underline{g}$ and $\mathscr{A} \subseteq[\mathbf{N}]^{\omega}$ is a nonfeeble family. Then $\mathscr{M}(\mathscr{A})$ is dominated by a set $\mathscr{D}$ of size $\underline{u}$.

The following two lemmas are implicit in [BL] but we include a proof here for completeness.

Lemma 6.8. Assume $\underline{u}<\underline{g}$. Let $\mathscr{A}$ be a nonfeeble family and $\mathscr{C}$ a family of less than $\underline{g}$ infinite subsets of $\mathbf{N}$. Then there is $r \in \mathbf{N} \uparrow \mathbf{N}$ such that $(\forall C \in$ $\mathscr{C})(\exists A \in \mathscr{A}) \quad \forall_{n}^{\infty} \operatorname{next}(C, n) \leq \operatorname{next}(A, r(n))$.

Proof. For $C \in \mathscr{C}$, define $\mathscr{G}(C)=\{Z \subseteq \mathbf{N}: Z$ is infinite and, for some $A \in \mathscr{A}, \operatorname{next}(C, n) \leq \operatorname{next}(A, \operatorname{next}(Z, n))$ for all sufficiently large $n\}$. We show that $\mathscr{G}(C)$ is groupwise dense. $\mathscr{G}(C)$ is clearly closed under subsets and finite modifications. So consider now an arbitrary partition of $\mathbf{N}$ into intervals $\left[p_{i}, p_{i+1}\right)$ : we show that $\mathscr{G}(C)$ contains the union of infinitely many of them.

By merging some intervals together, we can assume that each interval contains a member of $C$. Since $\mathscr{A}$ is nonfeeble, pick $A \in \mathscr{A}$ such that $A \cap$ $\left[p_{i}, p_{i+2}\right)=\varnothing$ for infinitely many $i \in \mathbf{N}$. Let $Z=\bigcup\left\{\left[p_{i}, p_{i+1}\right): A \cap\left[p_{i}, p_{i+2}\right)=\right.$ $\left.\varnothing \& A \cap\left[p_{i-1}, p_{i}\right) \neq \varnothing\right\}$. Then $Z$ is an infinite union of intervals; we verify that $\operatorname{next}(C, m) \leq \operatorname{next}(A, \operatorname{next}(Z, m))$ for all $m$. So fix $m$, say $m \in\left[p_{i}, p_{i+1}\right)$, and hence $\operatorname{next}(C, m) \leq p_{i+2}$ since $C$ meets each interval. Now $\operatorname{next}(Z, m) \in\left[p_{j}, p_{j+1}\right)$ for some $j \geq i$. By the definition of $Z$, we have $\operatorname{next}(A, \operatorname{next}(Z, m)) \geq p_{j+2} \geq p_{i+2} \geq \operatorname{next}(C, m)$ as desired.

This completes the proof that $\mathscr{G}(C)$ is groupwise dense for each $C \in \mathscr{C}$. Since $\mathscr{B}$ has size less than $\underline{g}$, pick $X \in \bigcap_{C \in \digamma} \mathscr{G}(C)$ and $r(n)=\operatorname{next}(Z, n)$ is the required function.

Lemma 6.9. Assume $\underline{u}<\underline{g}$. Let $\mathscr{A} \subseteq[\mathbf{N}]^{\omega}$ be a nonfeeble family and $\mathscr{U}$ an ultrafilter generated by $\underline{u}$ sets. Then there is a finite-to-one function $f$ such that

$$
\forall B \in \mathscr{U} \exists A \in \mathscr{A} \quad f[A] \subseteq^{*} f[B] .
$$


Proof. By using Lemma 6.8 on the $\underline{u}$ generators of $\mathscr{U}$, we can choose $r \in \mathbf{N} \uparrow \mathbf{N}$ such that

$$
\forall B \in \mathscr{U} \exists A \in \mathscr{A} \forall_{n}^{\infty} \quad \operatorname{next}(B, n) \leq \operatorname{next}(A, r(n)) .
$$

Define partition $\left[p_{i}, p_{i+1}\right)$ of $\mathbf{N}$ such that $r(n)<p_{i+2}$ whenever $n \in\left(p_{i}\right.$, $\left.p_{i+1}\right]$. For $i=0,1,2$, let $Z(i)=\bigcup_{n \in \mathrm{N}}\left[p_{3 n+i}, p_{3 n+i+1}\right)$. Since $\mathscr{U}$ is an ultrafilter, it must contain one of the sets $X(i)$, and for notational simplicity, we can assume that $Z(1) \in \mathscr{U}$.

Finally, define a finite-to-one function $f$ by $f(m)=n$ iff $m \in\left[p_{3 n}, p_{3(n+1)}\right)$. We claim that $f$ is as desired. Indeed, fix $B \in \mathscr{U}$ and assume without loss of generality that $B \subseteq Z(1)$. Choose $A \in \mathscr{A}$ such that $\forall_{n}^{\infty} \operatorname{next}(B, n) \leq$ $\operatorname{next}(A, r(n))$ and we show that $f[A] \subseteq^{*} f[B]$.

If $A \cap\left[p_{3 n}, p_{3(n+1)}\right) \neq \varnothing$ and $B \cap\left[p_{3 n}, p_{3(n+1)}\right)=\varnothing$, then $\operatorname{next}\left(A, r\left(p_{3 n-1}\right)\right) \leq$ $\operatorname{next}\left(A, p_{3 n}\right)<p_{3 n+3} \leq \operatorname{next}\left(B, p_{3 n-1}\right)$ since $B \subseteq Z(1)$. Hence this can happen only for finitely many $n$ and the proof is complete.

Now we conclude the proof of Proposition 6.7.

Let $\mathscr{A}$ be a nonfeeble family; we show that $\mathscr{M}(\mathscr{A})$ is dominated by a set $\mathscr{D}$ of size $\underline{u}$.

Let $\mathscr{U}$ be generated by $\underline{u}$ sets $\left\{C_{\alpha}: \alpha \leq \underline{u}\right\}$. By Lemma 6.9 , we find a nondecreasing finite-to-one function $g$ such that

$$
\forall B \in \mathscr{U} \exists A \in \mathscr{A} \quad g[A] \subseteq^{*} g[B] .
$$

The ultrafilter $g[\mathscr{U}]$ is generated by the family $\mathscr{B}=\left\{B_{\alpha}=g\left[C_{\alpha}\right]: \alpha \leq \underline{u}\right\}$. Call a set $B$ sparse if $b+1 \notin B$ whenever $b \in B$. The family $\mathscr{B}^{\prime}=\{B \in$ $\mathscr{B}: B$ is sparse $\}$ is still a base of $g[\mathscr{U}]$, so we may as well assume that every $B_{\alpha}$ is sparse in the first place. For $\alpha<\underline{u}$ and $n \in \mathbf{N}$ define $h_{\alpha}(n)=2^{z(\alpha, n)^{2}+1}$, where $z(\alpha, n)=\min g^{-1}\left\{\operatorname{next}\left(B_{\alpha}, g(n)\right)+1\right\}$. See Figure 6 .

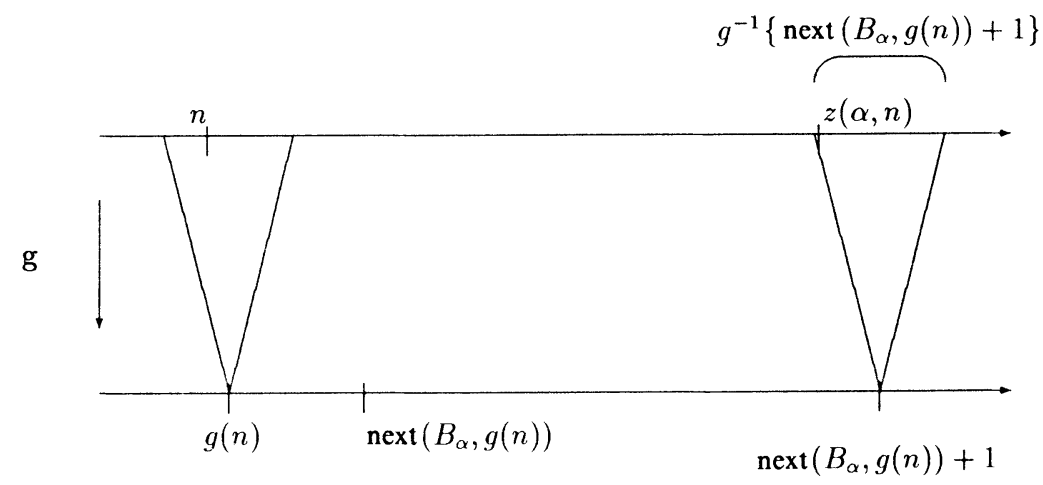

FiguRE 6 
We show that the family $\mathscr{D}=\left\{h_{\alpha}: \alpha<\underline{u}\right\}$ dominates $\mathscr{M}(\mathscr{A})$. Fix $f=$ $f_{\bar{I}} \in \mathscr{M}(\mathscr{A})$ where $\bar{I}=\left(I_{n}\right)_{n \in \mathbf{N}}$ is a cover of $\mathscr{M}(\mathscr{A})$. Put

$$
B=\left\{b \in \mathbf{N}: \sum_{k \geq b}\left|I_{k}\right| \geq 2^{-\left(\min g^{-1}\{g(b)+1\}\right)^{2}-1}\right\} .
$$

Claim 6.10. $g[B] \in g[\mathscr{U}]$.

Proof. It suffices to show that $g[B]$ meets each $B_{\alpha}$. Property (1) allows us to pick an $A \in \mathscr{A}$ so that $g[A] \subseteq^{*} B_{\alpha}$. Since $\mathscr{A} \subseteq[\mathbf{N}]^{\omega}$ and $B_{\alpha}$ is sparse, the set $A$ is both infinite and coinfinite. Hence, by Lemma 5.11,

$$
\exists^{\infty} a \in A \quad \sum_{k \geq a}\left|I_{k}\right| \geq 2^{-\operatorname{next}\left(A^{c}, a\right)^{2}-1} .
$$

Since $g[A] \subseteq^{*} B$ and $B$ is sparse,

$$
\forall^{\infty} a \in A \quad \operatorname{next}\left(A^{c}, a\right) \leq \min g^{-1}\{g(a)+1\},
$$

and hence $A \cap B$ is an infinite set. Now $g[A] \subseteq^{*} B_{\alpha}$ implies $g[B] \cap Y_{\alpha} \neq \varnothing$, as desired.

To complete the proof of Proposition 6.7, fix $\alpha$ so that $B_{\alpha} \subseteq g[B]$.

Notice that $\operatorname{next}(g(B), g(n)) \geq g(\operatorname{next}(B, n))$ for all $n$. So we may estimate:

$$
\begin{aligned}
h_{\alpha}(n) & =2^{z(\alpha, n)^{2}+1} \geq 2^{\left(\min g^{-1}\{\operatorname{next}(g(B), g(n))+1\}\right)^{2}+1} \\
& \geq 2^{\left(\min g^{-1}\{g(\operatorname{next}(B, n))+1\}\right)^{2}+1} \\
& \geq\left\lceil 1 / \sum_{k \geq \operatorname{next}(B, n)}\left|I_{k}\right|\right\rceil \geq\left\lceil 1 / \sum_{k \geq n}\left|I_{k}\right|\right\rceil \\
& =f_{\bar{I}}(n) .
\end{aligned}
$$

Hence $h_{\alpha}(n) \geq f_{\bar{I}}(n)$ for all $n$, as desired.

\section{THERE MAY BE MANY PAIRWISE INCOMPARABLE SETS}

Theorem 7.1. If there are $\kappa$ pairwise not cofinally equivalent ultrafilters on $\mathbf{N}$, then there exist $\kappa$ sets of measure zero, pairwise incomparable under the relation $\leq$.

By Theorem 1.4, if $\mathrm{CH}$ or MA holds, we get Figure 7.

In view of Corollary 5.4, Theorem 7.1 is an immediate consequence of the following.

Lemma 7.2. If $\mathscr{U}$ and $\mathscr{V}$ are ultrafilters that are not cofinally equivalent, then $\mathscr{S}(\mathscr{U})$ and $\mathscr{S}(\mathscr{V})$ are incomparable under the relation $\leq$.

Proof. Fix a sequence $\left(\varepsilon_{n}\right)_{n \in \mathbf{N}}$ of positive reals; we show that there are a set $A=$ $\{x(n): n \in \mathbf{N}\} \in \mathscr{U}$ and a cover $\bar{I}=\left(I_{n}\right)_{n \in \mathbf{N}}$ of $\mathscr{S}(\mathscr{V})$ such that $\sum_{k \geq x(n)}\left|I_{k}\right| \leq$ $\varepsilon_{x(n)}$ for each $n$. 


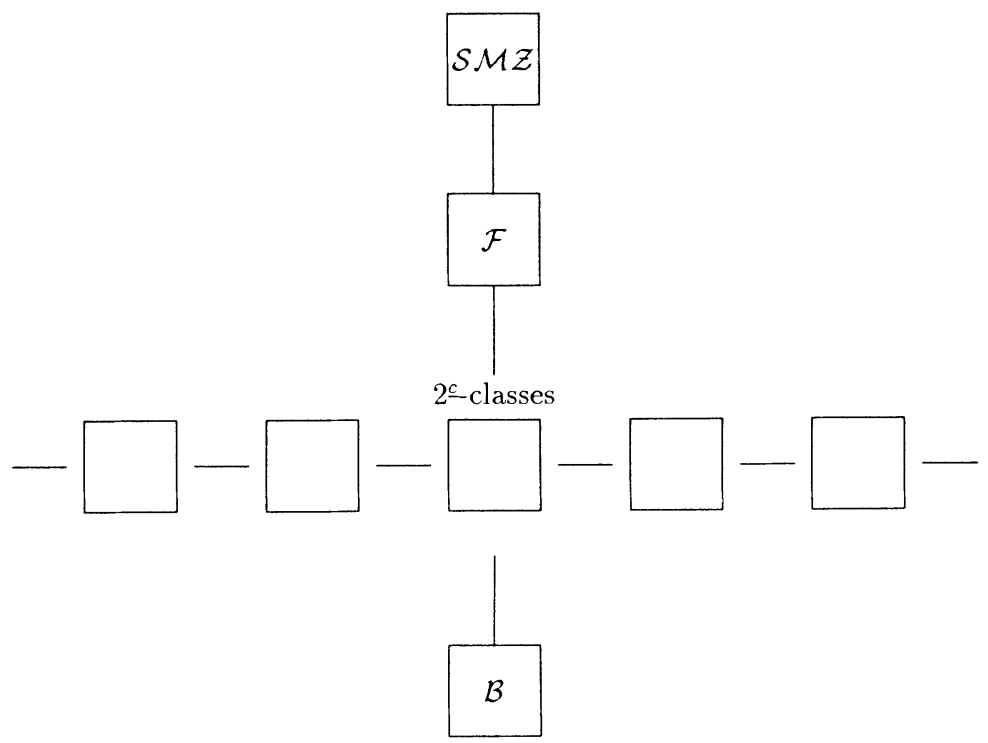

FIGURE 7

By Lemma 5.12 , this suffices to show that $\mathscr{S}(\mathscr{U}) \nsucceq \mathscr{S}(\mathscr{V})$; by symmetry $\mathscr{S}(\mathscr{V}) \nsucceq \mathscr{S}(\mathscr{U})$ and we shall be done. Without loss of generality we assume that $\varepsilon_{n} \geq \varepsilon_{n+1}$ for each $n$. Now define a partition of $\mathbf{N}$ into intervals $[a(n), a(n+1))$ as follows: We put $a(0)=0$, and given $a(n)$, we choose $a(n+1)$ large enough so that

$$
a(n+1) \geq \sum_{i \leq a(n)} 2^{i^{2}} \text { and } \sum_{k \geq a(n+1)} 2^{-2 k-1} \leq \varepsilon_{a(n)} .
$$

The following simple fact was proved in [Bs]:

Proposition 7.3. If the ultrafilters $\mathscr{U}$ and $\mathscr{V}$ are not cofinally equivalent, and $\mathbf{N}$ is partitioned into finite intervals, then there are $A \in \mathscr{U}$ and $B \in \mathscr{V}$ such that no union of two adjacent intervals from the partition intersects both $A$ and $B$.

Now let $A=\{x(n): n \in \mathbf{N}\} \in \mathscr{U}$ and $B=\{y(n): n \in \mathbf{N}\} \in \mathscr{V}$ be such that no interval $[a(n), a(n+2))$ meets both $A$ and $B$. Let $\bar{I}=\left(I_{n}\right)_{n \in \mathbf{N}}$ be the canonical cover of $\mathscr{S}(B)$. Since $\bar{I}$ is a cover of $\mathscr{S}(\mathscr{V})$ by 5.9 , we claim that $A$ and $\bar{I}$ are as desired. Indeed, if $x \in A$, say $x \in[a(n), a(n+1))$, then since $B \cap[a(n-1), a(n+2))=\varnothing$, we must have $\sum_{k \geq x}\left|I_{k}\right| \leq \sum_{k \geq a(n+2)} 2^{-2 k-1} \leq$ $\varepsilon_{a(n+1)} \leq \varepsilon$ as desired.

Corollary 7.4. If $\mathscr{S}(\mathscr{U}) \sim \mathscr{S}(\mathscr{V})$ for every two ultrafilters $\mathscr{U}$ and $\mathscr{V}$, then NCF holds.

Corollary 7.4 confirms a conjecture of J. Baumgartner. 


\section{UNCOUNTABLE CHAINS OF EQUIVALENCE CLASSES}

Theorem 8.1. Suppose there is a Q-point in $[\mathbf{N}]^{\omega}$, and $M A_{\kappa}(\sigma$-centered) holds. Then there is a sequence of isomorphic $Q$-points $\left(\mathscr{U}_{\xi}\right)_{\xi \in\left(\kappa^{+}\right)^{*}+\kappa^{+}}$such that $\mathscr{S}\left(\mathscr{U}_{\xi}\right)$ $<\mathscr{S}\left(\mathscr{U}_{\xi}\right)$ for $\xi>\eta$.

Figure 8 emerges:

Corollary 8.2. Suppose that there is a $Q$-point in $[\mathbf{N}]^{\omega}$. Then there exists a sequence $\left(\mathscr{U}_{\xi}\right)_{\xi \in \omega_{1}^{*}+\omega_{1}}$ of isomorphic Q-points such that $\mathscr{S}\left(\mathscr{U}_{\xi}\right)>\mathscr{S}\left(\mathscr{U}_{\eta}\right)$ for $\xi>\eta$.

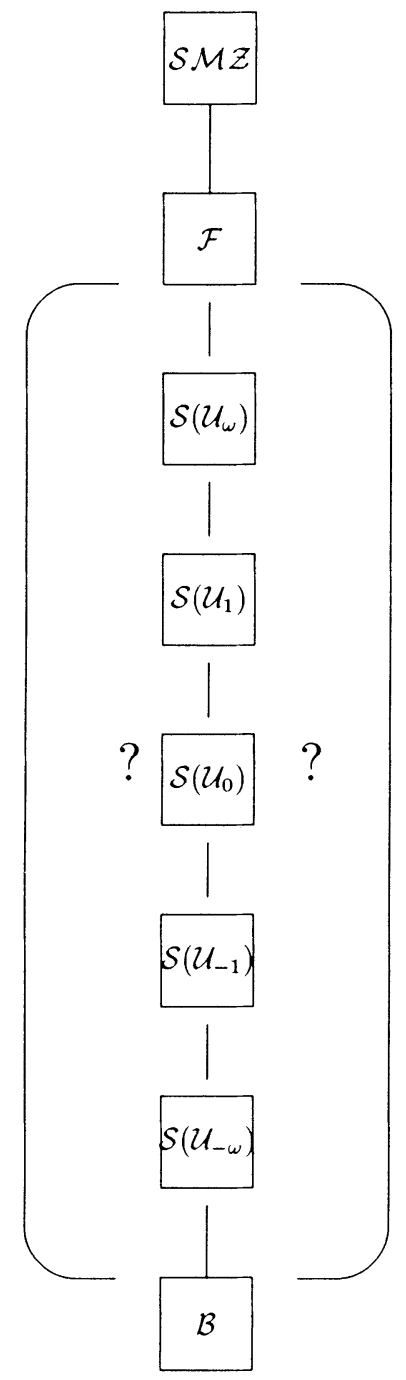

Figure 8 
Proof. Notice that $\mathrm{MA}_{\omega}$ is a theorem of ZFC.

Proof of Theorem 8.1. Throughout this section, we let $p_{j}=2^{j^{2}+1}$ for each $j \in \mathbf{N}$.

Lemma 8.3. Suppose $\mathscr{V}$ is a Q-point and $F$ is a nondecreasing finite-to-one function mapping $\mathbf{N}$ onto $\mathbf{N}$ such that

$$
\forall_{n}^{\infty} \exists_{j}\left[p_{j}, p_{j+2}\right) \subseteq F^{-1}\{n\} .
$$

Then $\mathscr{S}(\mathscr{U})>\mathscr{S}(\mathscr{V})$ where $\mathscr{U}=F[\mathscr{V}]$.

We need to prove that $\mathscr{S}(\mathscr{U}) \geq \mathscr{S}(\mathscr{V})$ and $\mathscr{S}(\mathscr{V}) \nsubseteq \mathscr{S}(\mathscr{U})$; hence we break the proof of Lemma 8.3 into two parts.

I: $\mathscr{S}(\mathscr{U}) \geq \mathscr{S}(\mathscr{U})$. Define a function $h:(0,1) \rightarrow \mathbf{N}$ by $h(x)=\max \{m \in$ $\left.\mathbf{N}: x \leq 2^{-2 m}\right\}$. The function $h$ has the following property:

(P) If $\bar{J}=\left(J_{n}\right)_{n \in \mathbf{N}}$ is the canonical cover of $\mathscr{S}(\{\mathscr{A}\})$ where $A \subseteq$ $\mathbf{N}$ is infinite and $x=\sum_{k \geq n}\left|J_{k}\right|$ for some $n$, then $A \cap[s(n)$, $h(x)) \neq \varnothing$ where $s(n)=\min \left\{m \in \mathbf{N}: \sum_{k=0}^{m} 2^{k^{2}} \geq n\right\}$.

Now define $t: \mathbf{N} \rightarrow(0,1)$ by $t(m)=\min \left\{2^{-(n+1)^{2}-1}: F(n)<m\right\}$. Observe that $t$ is well defined since $F$ is finite-to-one. Finaly, define $r:(0,1) \rightarrow(0,1)$ by $r(x)=t(h(x))$. This function is nondecreasing. We verify that it witnesses $\mathscr{S}(\mathscr{U}) \geq \mathscr{S}(\mathscr{V})$.

Fix a cover $\bar{I}=\left(I_{n}\right)_{n \in \mathrm{N}}$ of $\mathscr{S}(\mathscr{V})$. By Lemma 5.11, there exists $B \in \mathscr{V}$ such that $\sum_{k \geq y}\left|I_{k}\right| \geq 2^{-(y+1)^{2}-1}$ for each $y \in B$. Let $A=F[B] \in \mathscr{U}$ and $\bar{J}=\left(J_{n}\right)_{n \in \mathbf{N}}$ be the canonical cover of $\mathscr{S}(\{A\})$. We check that $r\left(\sum_{k \geq n}\left|J_{k}\right|\right) \leq$ $\sum_{k \geq n}\left|I_{k}\right|$ for almost all $n$.

Fix $n$ and put $z=\sum_{k \geq n}\left|J_{k}\right|$. By property (P) of the function $h$, there exists $x \in A \cap[s(n), h(z))$. Pick any $y \in B \cap F^{-1}\{x\}$. But now, $r(z)=t(h(z)) \leq$ $2^{-(y+1)^{2}-1} \leq \sum_{k \geq y}\left|I_{k}\right|$ by the choice of $B$ and definition of $r$. Hence, we obtain the desired inequality if $y \geq n$. Moreover, for almost all $k$ we can pick $j(k)$ such that $F^{-1}\{k\} \supseteq\left[p_{j(k)}, p_{j(k)+2}\right)$ and hence $k \leq j(k)$ holds for almost all $k$. We conclude that if $s(n)$ is large enough, then

$$
n \leq \sum_{k=0}^{s(n)} 2^{k^{2}} \leq \sum_{k=0}^{x} 2^{k^{2}} \leq 2^{(x-1)^{2}}=2^{(x-1+2)^{2}} \leq 2^{(j(x-1)+2)^{2}} \leq y
$$

as desired.

II: $\mathscr{S}(\mathscr{V}) \nsupseteq \mathscr{S}(\mathscr{U})$. It suffices to show that for each sequence $\left(\varepsilon_{n}\right)_{n \in \mathbf{N}}$ of positive reals, there are sets $A=\left(x_{n}\right)_{n \in \mathbf{N}} \in \mathscr{V}$ and $B=\left(y_{n}\right)_{n \in \mathbf{N}} \in \mathscr{U}$ such that:
(1) $\forall_{n}^{\infty} \quad x_{n+1} \geq x_{n}+2^{y_{n+1}^{2}}$;
(2) $\forall_{n}^{\infty} \quad \sum_{k \geq y_{n+1}} 2^{-2 k-1} \leq x_{n}$. 
Indeed, because $\mathscr{U}$ and $\mathscr{V}$ are ultrafilters we could assume that (1) and (2) hold for each $n$ and then the canonical cover of $\mathscr{S}(\{B\})$ would satisfy the hypothesis of Lemma 5.12 and hence the result would follow.

So fix such a sequence $\left(\varepsilon_{n}\right)_{n \in \mathbf{N}}$ and assume without loss of generality that $\varepsilon_{n} \geq \varepsilon_{n+1}$ for each $n$. Pick $m$ large enough so that $F^{-1}\{n\} \supseteq\left[p_{j(n)}, p_{j(n)+1}\right)$ for each $n \geq m$. Now define a sequence $\left(a_{k}\right)_{k \in \mathbf{N}}$ by $a_{0}=p_{j(m+1)}$ and, given $a_{k}$, choose $a_{k+1}$ large enough so that

(i) $F\left(a_{k+1}\right)>a_{k}$.

(ii) $\sum_{i \geq a_{k+1}} 2^{-2 i-1} \leq \varepsilon_{a_{k}}$.

Since $\mathscr{V}$ is an ultrafilter, there is an $i \in\{0,1,2\}$ such that

$$
A_{i}=\bigcup_{k \in \mathbf{N}}\left[a_{3 k+i}, a_{3 k+i+1}\right) \in \mathscr{V} ;
$$

assume for notational simplicity that $A_{0} \in \mathscr{V}$ and because $\mathscr{V}$ is a $Q$-point, pick $A \subseteq A_{0}$ in $\mathscr{V}$ such that $\left|A \cap\left[a_{3 k}, a_{3 k+1}\right)\right| \leq 1$ for each $k$. Put $B=F[A] \in$ $\mathscr{U}$ and enumerate both sets as $A=\left\{x_{n}: n \in \mathbf{N}\right\}$ and $B=\left\{y_{n}: n \in \mathbf{N}\right\}$. For each $n, x_{n}+2^{y_{n+1}} \leq 2^{y_{n+1}^{2}+1}$ since $x_{n} \leq y_{n+1}$. Now if $z=y_{n+1}-1$ and since $z \leq j(z)$ for almost all $z$, we have $2^{y_{n+1}^{2}+1}=2^{(z+1)^{2}+1} \leq 2^{(j(z)+1)^{2}+1} \leq x_{n+1}$ for almost all $n$.

Moreover, if $x_{n} \in\left[a_{3 k}, a_{3 k+1}\right)$, then $y_{n+1}=f\left(x_{n+1}\right) \geq a_{3 k+2}$ and hence

$$
\sum_{i \geq y_{n+1}} 2^{-2 i-1} \leq \sum_{i \geq a_{3 k+2}} 2^{-2 i-1} \leq \varepsilon_{a_{3 k+1}} \leq \varepsilon_{x_{n}}
$$

and this completes the proof of Lemma 8.3.

Definition 8.4. Let $\bar{N}=\left(N_{j}\right)_{j \in \mathbf{N}}$ and $\bar{M}=\left(M_{i}\right)_{i \in \mathbf{N}}$ be partitions of $\mathbf{N}$ into disjoint consecutive intervals. We write $\bar{M}>\bar{N}$ and say that $\bar{M}$ is coarser than $\bar{N}$ if $\forall_{i}^{\infty} \exists j(i), j(i+1) \quad M_{i}=\bigcup_{j=j(i)}^{j(i+1)-1} N_{j}$ and

$$
\forall_{i}^{\infty} \exists w(i) j(i) \leq 2^{w(i)^{2}+1}<2^{(w(i)+2)^{2}+1}<j(i+1) .
$$

The relation $>$ is a strict partial order.

Lemma 8.5. Let $\mathcal{N}$ be a family of partitions of $\mathbf{N}$ into disjoint consecutive intervals, upward directed by the relation $>$. Then there exist a $\sigma$-centered forcing notion $\mathbf{P}$ and a family $\mathscr{D}$ of dense subsets of $\mathbf{P}$ such that $|\mathscr{D}| .=|\mathcal{N}|+\aleph_{0}$ and if $G$ is a filter in $\mathbf{P}$ which meets each $D \in \mathscr{D}$, then there exists a partition $\bar{M}$. coarser than each partition in $\mathcal{N}$.

Proof. Define

$$
\begin{array}{r}
\mathbf{P}=\{(s, \sigma): s \text { is a function defined on a set }\{0, \ldots, k-1\} \\
\text { such that } s(0), \ldots, s(k-1) \text { are consecutive disjoint } \\
\text { intervals of } \mathbf{N}, \text { and } \sigma \subseteq \mathscr{N} \text { is a finite subfamily }\} .
\end{array}
$$


We define $\left(s^{\prime}, \sigma^{\prime}\right) \leq(s, \sigma)$ if $s^{\prime}$ extends $s, \sigma \subseteq \sigma^{\prime}$ and if $\bar{N} \in \sigma, i \in$ $\operatorname{dom}\left(s^{\prime}\right) \backslash \operatorname{dom}(s)$ then $s(i)=[k, n)$ where $n$ is the endpoint of an interval of $\bar{N}$ and there is $w^{*}$ such that $N_{j} \subseteq[k, n)$ for all $2^{\left(w^{*}\right)^{2}+1} \leq j \leq 2^{\left(w^{*}+i\right)^{2}+1}$.

$\mathbf{P}$ is $\sigma$-centered since $\left(s^{\prime}, \sigma^{\prime}\right)$ and $(s, \sigma)$ are compatible whenever $s=s^{\prime}$. The required family of dense sets consists of the sets $D_{i}=\{(s, \sigma): i \in \operatorname{dom}(s)\}$ for $i \in \mathbf{N}$ and $D_{\bar{N}}=\{(s, \sigma): \bar{N} \in \sigma\}$ for $\bar{N} \in \mathcal{N}$.

Corollary 8.6. If $M A_{\kappa}(\sigma$-centered $)$ holds, and there exists a Q-point, then there exists a strictly increasing chain of type $\kappa^{+}$of more and more rarefied sets of the form $\mathscr{S}\left(\mathscr{U}_{\xi}\right)$, where $U_{\eta}$ is the image of $U_{\xi}$ via a finite-to-one function mapping $\mathbf{N}$ onto $\mathbf{N}$ whenever $\xi<\eta$.

Before we show how to get uncountable descending sequences of equivalence classes, we want to mention the following fact which shows that all $Q$-points constructed in this section are indeed isomorphic.

Proposition 8.7. If $\mathscr{V}$ is a $Q$-point and $F$ a finite-to-one function such that $\mathscr{U}=F[\mathscr{V}]$, then $\mathscr{U}$ and $\mathscr{V}$ are isomorphic.

Proof. Since $\mathscr{V}$ is a $Q$-point, there exists a $B \in \mathscr{V}$ such that $F \mid B$ is oneto-one, and $F[B]$ is coinfinite in $\mathbf{N}$. It is not hard to see that any permutation of $\mathbf{N}$ that extends $F \mid B$ witnesses the isomorphism between $\mathscr{V}$ and $\mathscr{U}$.

For strictly increasing functions $f, g: \mathbf{N} \rightarrow \mathbf{N}$ we write in this section $f<^{*}$ $g$ if $\forall_{n}^{\infty} f(n)<g(n)$. (Notice that this is somewhat stronger than $f \leq^{*} g$ and $g \not^{*} f$ as defined in the introduction.)

It is well known that $\mathrm{MA}_{\kappa}\left(\sigma\right.$-centered) implies the existence of a $<^{*}$-well ordered chain of order type $\kappa^{+}$and it is also known that it is relatively consistent with the negation of $\mathrm{CH}$ that no such chain of order type $\omega_{2}$ exists.

It follows that the next lemma is the last ingredient needed for the proof of Theorem 8.1.

Lemma 8.8. Suppose there exist a $Q$-point $\mathscr{U}$ and a sequence $\left(f_{\xi}\right)_{\xi<\lambda}$ of strictly increasing functions mapping $\mathbf{N}$ into $\mathbf{N}$ such that $f_{\xi}<{ }^{*} f_{\eta}$ for all $\xi<\eta<\lambda$. Then there exists a sequence $\left(\mathscr{U}_{\xi}\right)_{\xi<\lambda}$ of Q-points such that $\mathscr{S}\left(\mathscr{U}_{\xi}\right)>\mathscr{S}\left(\mathscr{U}_{\eta}\right)$ for $\xi<\eta<\lambda$.

Proof. Fix $\left(f_{\xi}\right)_{\xi<\lambda}$ and $\mathscr{U}$ as in the hypothesis of the lemma, and let $\left(G_{i, j}\right)_{i<j<\omega}$ be a sequence of nondecreasing, finite-to-one functions mapping $\mathbf{N}$ onto $\mathbf{N}$ such that

(i) $G_{i, j} \circ G_{j, k}(n)=G_{i, k}(n)$ for all $i<j<k$ and $n$, and

(ii) $\forall n \quad \forall i<j \quad \exists r \quad G_{i, j}^{-1}\{n\} \supset\left[p_{r}, p_{r+4}\right)$.

For $\xi<\lambda$, let $H_{\xi}$ be the only nondecreasing function mapping $\mathbf{N}$ onto $\mathbf{N}$ such that $\left|H_{\xi}^{-1}\{n\}\right|=\left|G_{0, f_{\xi}(n)}^{-1}\{n\}\right|$ for all $n$. Moreover, let $\mathscr{U}_{\xi}$ be the filter generated by the family $\left\{\bigcup_{n \in A} H_{\xi}^{-1}\{n\}: A \in \mathscr{U}\right\} \cup\left\{\left\{\min \left(H_{\xi}^{-1}\{n\}\right): n \in \mathbf{N}\right\}\right\}$. Claim 8.9. For all $\xi$, the filter $\mathscr{U}_{\xi}$ is a $Q$-point and $H_{\xi}\left[\mathscr{U}_{\xi}\right]=\mathscr{U}$. 
Proof. The second part is obvious. To see that $\mathscr{U}_{\xi}$ is a $Q$-point, let $\left(\left[q_{i}, q_{i+1}\right)\right)_{i \in \mathbf{N}}$ be a partition of $\mathbf{N}$ into pairwise disjoint intervals. For every $i$, let $\left[r_{i}, r_{i+1}\right)=$ $\left\{n: \min \left(H_{\xi}^{-1}\{n\}\right) \in\left[q_{i}, q_{i+1}\right)\right\}$. Some of the intervals $\left[r_{i}, r_{i+1}\right)$ may be empty, but this is none of our concern. Let $A \in \mathscr{U}$ be such that $\left|A \cap\left[r_{i}, r_{i+1}\right)\right| \leq 1$ for all $i$. Then $B=\left\{\min \left(H^{-1}\{n\}\right): n \in A\right\} \in \mathscr{U}_{\xi}$, and $\left|B \cap\left[q_{i}, q_{i+1}\right)\right| \leq 1$ for all $i$.

The following claim is the last brick needed for the proof of Lemma 8.8 and hence Theorem 8.1.

Claim 8.10. Let $\xi<\eta<\lambda$. Then there is a nondecreasing finite-to-one function $H_{\xi, \eta}$ that maps $\mathbf{N}$ onto $\mathbf{N}$ such that $H_{\xi, \eta}\left[\mathscr{U}_{\eta}\right]=\mathscr{U}_{\xi}$, and $H_{\xi, \eta}$ satisfies condition $(*)$ of Lemma 8.3.

Proof. Fix $\xi<\eta<\lambda$, and let $k_{0}$ be such that $f_{\eta}(k)>f_{\xi}(k)$ and $\min \left(H_{\xi}^{-1}\{k\}\right)$ $<\min \left(H_{\eta}^{-1}\{k\}\right)$ for all $k \geq k_{0}$. It is not hard to see that $k_{0}$ as above exists.

Let $n_{0}=\min \left(H_{\eta}^{-1}\left\{k_{0}\right\}\right)$ and for each $n \geq n_{0}$ denote $H_{\eta}(n)=m, f_{\xi}(m)=$ $i, f(m)=j>i$. Suppose $n$ is the $k$ th element of $H_{\eta}^{-1}\{m\}$. Then we put

$$
\begin{aligned}
H_{\xi, \eta}(n)= & G_{i, j}\left(\max \left(G_{0, j}^{-1}\{m-1\}\right)+k\right) \\
& -\max \left(G_{0,1}^{-1}\{m-1\}\right)+\max \left(H_{\xi}^{-1}\{m-1\}\right) .
\end{aligned}
$$

For $n<n_{0}$ we define $H_{\xi, \eta}(n)$ in such a way that $H_{\xi, \eta}$ is nondecreasing and maps $\mathbf{N}$ onto $\mathbf{N}$. This is possible, since $H_{\xi, \eta}\left(n_{0}\right)=\min \left(H_{\xi}^{-1}\left\{k_{0}\right\}\right) \leq n_{0}$. Notice that for $n \geq n_{0}$ the number $H_{\xi, \eta}(n)$ was chosen in such a way that $H_{\xi} \circ H_{\xi, \eta}(n)=H_{\eta}(n)$, and if

$$
A=\left\{\min \left(H_{\eta}^{-1}\{m\}\right): m \in \mathbf{N}\right\} \quad \text { and } \quad B=\left\{\min \left(H^{-1}\{m\}\right): m \in \mathbf{N}\right\},
$$

then $H_{\xi, \eta}\left[A \backslash n_{0}\right]=B \backslash H_{\xi, \eta}\left(n_{0}\right)$.

By the definition of $\mathscr{U}_{\xi}$, we have therefore $H_{\xi, \eta}\left[\mathscr{U}_{\eta}\right]=\mathscr{U}_{\xi}$. Now observe that if $i, j, k, m, n$ are as above, then since $f_{\xi}$ and $f_{\eta}$ are increasing functions we have

$$
\min \left(G_{0, i}^{-1}\{m\}\right) \geq \min \left(H_{\xi}^{-1}\{m\}\right) \quad \text { and } \min \left(G_{0, j}^{-1}\{m\}\right) \geq \min \left(H_{\eta}^{-1}\{m\}\right) .
$$

If $H_{\xi, \eta}(n)=t$, then $\left|H_{\xi, \eta}^{-1}\{t\}\right|=\left|G_{i, j}^{-1}\{s\}\right|$, where

$$
s=G_{i, j}\left(\max \left(G_{0, j}^{-1}\{m-1\}\right)+k\right) .
$$

By what we observed above, $\max \left(H_{\xi}^{-1}\{m-1\}\right)-\max \left(G_{0, i}^{-1}\{m-1\}\right)<0$, so $\min \left(H_{\xi, \eta}^{-1}\{t\}\right)<\min \left(G_{i, j}^{-1}\{s\}\right)$. Now it is not hard to see that since $G_{i, j}^{-1}\{s\}$ contains by (ii) a set of the form $\left[p_{r}, p_{r+4}\right)$, the set $H_{\xi, \eta}^{-1}(t)$ must contain a set of the form $\left[p_{j}, p_{j+2}\right)$ and the proof is complete. 


\section{OPEN PROBLEMS}

The most tantalizing question is perhaps

Question 1. Is is consistent that there are only four equivalence classes of the relation " $\sim$ "? In particular, does $\underline{u}<\underline{g}$ imply the existence of only four classes?

A more general problem is

Question 2. Which are the possible cardinalities of the family of equivalence classes of the relation " $\sim$ "?

We have seen that $2^{\underline{c}}$ is a possibility, and in view of the results of $\S 6$, it seems quite likely that some finite values are possible. Perhaps one could rule out $\omega, \omega_{1}$, or $\underline{c}$ ? But we don't even know the answer to the following:

Question 3. Is is consistent that the equivalence classes of the relation " " are linearly ordered?

Conjecture 4. If $X<\mathscr{S}(\mathscr{U})$ for every ultrafilter $\mathscr{U}$, then $X \in \mathscr{B}$.

Conjecture 5. If $X$ is a Borel $\left(\Sigma_{1}^{1}\right)$ set of measure zero, then $X$ is in $\mathscr{S} \mathscr{M} \mathscr{Z}$, $\mathscr{F}$, or $\mathscr{B}$.

We conclude this section with a problem that already came up in other contexts (see [Bl and $\mathrm{BL}]$ ).

Question 6. Does NCF imply that $\underline{u}<\underline{g}$ ?

Notice that in $\S 6$, we use the full force of the inequality $\underline{u}<\underline{g}$, whereas in $\S 7$ it was shown that unless NCF holds, there are ultrafilters $\mathscr{U}$ and $\mathscr{V}$ such that $\mathscr{S}(\mathscr{U})$ and $\mathscr{S}(\mathscr{V})$ are not equivalent.

Added in proof. The second author has answered both Questions 1 and 2 positively.

\section{REFERENCES}

[B] E. Borel, Eléments de la théorie des ensembles, Editions Albin Michel, Paris, 1949.

[BI] A. Blass, Applications of superperfect forcing and its relatives, Proceedings of the Set Theory Conference, York University, 1987 (to appear).

[Bs] _ Near coherence of filters. II, Trans. Amer. Math. Soc. 300 (1987), 557-581.

[BL] A. Blass and C. Laflamme, Consistency results about filters and the number of equivalent growth types, to appear.

[CN] W. Comfort and S. Negrepontis, The theory of ultrafilters, Springer-Verlag, New York, 1974.

[F] M. Fréchet, Les probabilités nulles et la raréfaction, Ann. Sci. École Norm. Sup. (3) 80 (1963), 139-172. 
[Lf] C. Laflamme, Sur la raréfaction des ensembles de mesure nulle, preprint, University of Toronto.

[O] J. Oxtoby, Measure and category, Springer-Verlag, New York, 1971.

Department of Mathematics, University of Toronto, Toronto, Ontario, Canada M5S 1A1

Institute of Mathematics, University of Warsaw, 00-901 Warsaw, Poland

Current address (Winifried Just): Mathematical Sciences Research Institute, 1000 Centennial Drive, Berkeley, California 94720

Current address (Claude Laflamme): Department of Mathematics, York University, North York, Ontario, Canada M3J 1P3 